Supplement of

\title{
Responses of Arctic black carbon and surface temperature to multi-region emission reductions: a Hemispheric Transport of Air Pollution Phase 2 (HTAP2) ensemble modeling study
}

Na Zhao et al.

Correspondence to: Kan Huang (huangkan@fudan.edu.cn) and Joshua S. Fu (jsfu@utk.edu)

The copyright of individual parts of the supplement might differ from the article licence. 


\section{S1 Statistical metrics of the model evaluation}

34 The statistical metrics in the present analysis include values of mean bias (MB), mean absolute error 35 (MAE), normalized mean bias (NMB), normalized mean error (NME), and correlative coefficient (R).

36 Detail equations of the above statistical metrics are shown as follows:

$37 \quad \mathrm{MB}=\frac{1}{\mathrm{~N}} \sum_{\mathrm{i}=1}^{\mathrm{N}}(\mathrm{Cm}-\mathrm{Co})$

$38 \quad \mathrm{MAE}=\frac{1}{\mathrm{~N}} \sum_{\mathrm{i}=1}^{\mathrm{N}}|\mathrm{Cm}-\mathrm{Co}|$

$39 \mathrm{NMB}=\frac{\sum_{\mathrm{i}=1}^{\mathrm{N}}(\mathrm{Cm}-\mathrm{Co})}{\sum_{\mathrm{i}=1}^{\mathrm{N}} \mathrm{Co}} \times 100 \%$

$40 \quad \mathrm{NME}=\frac{\sum_{\mathrm{i}=1}^{\mathrm{N}}|\mathrm{Cm}-\mathrm{Co}|}{\sum_{\mathrm{i}=1}^{\mathrm{N}} \mathrm{Co}} \times 100 \%$

$41 \quad \mathrm{COR}=\frac{\sum_{\mathrm{i}=1}^{\mathrm{N}}(\mathrm{Cm}-\overline{\mathrm{Cm}})(\mathrm{Co}-\overline{\mathrm{Co}})}{\sqrt{\sum_{\mathrm{i}=1}^{\mathrm{N}}(\mathrm{Cm}-\overline{\mathrm{Cm}})^{2} \sum_{\mathrm{i}=1}^{\mathrm{N}}(\mathrm{Co}-\overline{\mathrm{Co}})^{2}}}$

42 Where:

$43 \quad \mathrm{Cm}=$ The model-estimated concentration at station $i$, month $j$

$44 C o=$ The observed concentration at station $i$, month $j$

$45 \overline{\mathrm{Cm}}=$ The average model-estimated concentration of all hours of all sites

$46 \overline{C o}=$ The average observed concentration of all hours of all sites

$47 \quad N=$ The total numbers of hours of all sites for which the simulations are compared against observations

48

49 References

50 United States Environmental Protection Agency (U.S. EPA). Guidance on the use of models and other analyses for demonstrating attainment of air quality goals for ozone, $\mathrm{PM}_{2.5}$, and regional haze. U.S. Environmental Protection Agency Office of Air Quality Planning and Standards Air Quality Analysis Division Air Quality Modeling Group Research Triangle Park, NC. 2007.

U.S. EPA. Guidance on the Development, Evaluation, and Application of Environmental Models. Council for Regulatory Environmental Modeling U.S. Environmental Protection Agency Washington, DC. 2009. 


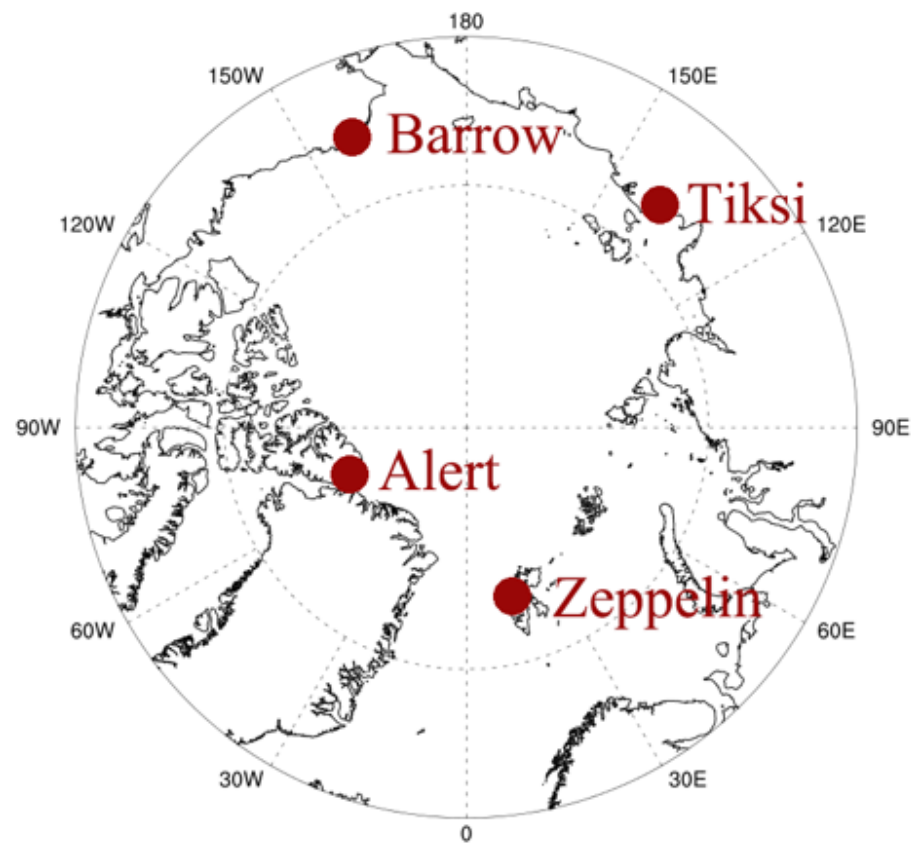

57 Figure S1 Four BC monitoring sites (Alert, Barrow, Tiksi, and Zeppelin) in the Arctic Circle. 
58 Table S1 Comparison of the simulations of BC concentrations of participating models at (a) Alert,

59 (b) Barrow, (c) Tiksi, and (d) Zeppelin in 2010.

60 (a) Alert

\begin{tabular}{|c|c|c|c|c|c|c|}
\hline Parameters & & CAMchem & CHASER_re1 & GEOSCHEM & GOCART-v5 & Oslo CTM3-v2 \\
\hline \multirow{5}{*}{$\mathrm{COR}^{\mathrm{a}}$} & CAMchem & - & 0.81 & 0.80 & 0.86 & 0.65 \\
\hline & CHASER_re1 & 0.81 & - & 0.61 & 0.70 & 0.86 \\
\hline & GEOSCHEM & 0.80 & 0.61 & - & 0.98 & 0.44 \\
\hline & GOCART-v5 & 0.86 & 0.70 & 0.98 & - & 0.48 \\
\hline & Oslo CTM3-v2 & 0.65 & 0.86 & 0.44 & 0.48 & - \\
\hline \multirow{5}{*}{$\begin{array}{c}\mathrm{NMB}^{\mathrm{b}} \\
(\%)\end{array}$} & CAMchem & - & -92.99 & -74.12 & -81.17 & 97.44 \\
\hline & CHASER_re1 & 1642.07 & - & 346.18 & 217.66 & 2785.04 \\
\hline & GEOSCHEM & 370.56 & -69.26 & - & -27.08 & 786.33 \\
\hline & GOCART-v5 & 562.46 & -57.38 & 40.04 & - & 1158.33 \\
\hline & Oslo CTM3-v2 & -33.38 & -96.10 & -82.90 & -87.57 & - \\
\hline \multirow{5}{*}{$\begin{array}{c}\mathrm{NME}^{\mathrm{c}} \\
(\%)\end{array}$} & CAMchem & - & 92.99 & 74.12 & 81.17 & 106.21 \\
\hline & CHASER_re1 & 1642.07 & - & 346.18 & 217.66 & 2785.04 \\
\hline & GEOSCHEM & 370.56 & 69.26 & - & 27.08 & 786.33 \\
\hline & GOCART-v5 & 562.46 & 57.38 & 40.04 & - & 1158.33 \\
\hline & Oslo CTM3-v2 & 45.70 & 96.10 & 82.90 & 87.57 & - \\
\hline \multirow{5}{*}{$\begin{array}{c}\mathrm{MB}^{\mathrm{d}} \\
\left(\mathrm{ng} \mathrm{m}^{-3}\right)\end{array}$} & CAMchem & - & -34.74 & -7.03 & -11.63 & 1.07 \\
\hline & CHASER_re1 & 34.74 & - & 27.71 & 23.12 & 35.81 \\
\hline & GEOSCHEM & 7.03 & -27.71 & - & -4.59 & 8.10 \\
\hline & GOCART-v5 & 11.63 & -23.12 & 4.59 & - & 12.69 \\
\hline & Oslo CTM3-v2 & -1.07 & -35.81 & -8.10 & -12.69 & - \\
\hline \multirow{5}{*}{$\begin{array}{c}\mathrm{MAE}^{\mathrm{e}} \\
\left(\mathrm{ng} \mathrm{m}^{-3}\right)\end{array}$} & CAMchem & - & 34.74 & 7.03 & 11.63 & 1.22 \\
\hline & CHASER_re1 & 34.74 & - & 27.71 & 23.12 & 35.81 \\
\hline & GEOSCHEM & 7.03 & 27.71 & - & 4.59 & 8.10 \\
\hline & GOCART-v5 & 11.63 & 23.12 & 4.59 & - & 12.69 \\
\hline & Oslo CTM3-v2 & 1.22 & 35.81 & 8.10 & 12.69 & - \\
\hline
\end{tabular}

61

62 (b) Barrow

\begin{tabular}{ccccccc}
\hline Parameters & & CAMchem & CHASER_re1 & GEOSCHEM & GOCART-v5 & Oslo CTM3-v2 \\
\hline & CAMchem & - & 0.91 & 0.90 & 0.89 & 0.84 \\
\cline { 2 - 7 } COR $^{\mathrm{a}}$ & CHASER_re1 & 0.91 & - & 0.82 & 0.79 & 0.81 \\
\cline { 2 - 7 } & GEOSCHEM & 0.90 & 0.82 & - & 0.90 & 0.92 \\
\cline { 2 - 7 } & GOCART-v5 & 0.89 & 0.79 & 0.90 & - & 0.71 \\
\cline { 2 - 7 } & Oslo CTM3-v2 & 0.84 & 0.81 & 0.92 & 0.71 & - \\
\hline \multirow{2}{*}{ NMB $^{\mathrm{b}}$} & CAMchem & - & -75.42 & -3.89 & -34.61 & 137.53 \\
\cline { 2 - 7 }$(\%)$ & CHASER_re1 & 399.17 & - & 310.46 & 188.49 & 854.96 \\
\cline { 2 - 7 } & GEOSCHEM & 49.47 & -69.06 & - & -26.62 & 165.92 \\
\hline
\end{tabular}




\begin{tabular}{|c|c|c|c|c|c|c|}
\hline & GOCART-v5 & 99.71 & -55.97 & 45.54 & - & 305.41 \\
\hline & Oslo CTM3-v2 & -38.57 & -87.62 & -54.76 & -66.86 & - \\
\hline \multirow{5}{*}{$\begin{array}{c}\mathrm{NME}^{\mathrm{c}} \\
(\%)\end{array}$} & CAMchem & - & 75.42 & 47.82 & 42.42 & 141.75 \\
\hline & CHASER_re1 & 399.17 & - & 310.46 & 190.71 & 854.96 \\
\hline & GEOSCHEM & 75.31 & 69.06 & - & 27.32 & 165.92 \\
\hline & GOCART-v5 & 105.96 & 58.53 & 46.22 & - & 305.41 \\
\hline & Oslo CTM3-v2 & 44.22 & 87.62 & 54.76 & 66.86 & - \\
\hline \multirow{5}{*}{$\begin{array}{c}\mathrm{MB}^{\mathrm{d}} \\
\left(\mathrm{ng} \mathrm{m}^{-3}\right)\end{array}$} & CAMchem & - & -37.49 & -3.04 & -11.02 & 8.27 \\
\hline & CHASER_re1 & 37.49 & - & 34.45 & 26.47 & 45.76 \\
\hline & GEOSCHEM & 3.04 & -34.45 & - & -7.98 & 11.31 \\
\hline & GOCART-v5 & 11.02 & -26.47 & 7.98 & - & 19.29 \\
\hline & Oslo CTM3-v2 & -8.27 & -45.76 & -11.31 & -19.29 & - \\
\hline \multirow{5}{*}{$\begin{array}{c}\mathrm{MAE}^{\mathrm{e}} \\
\left(\mathrm{ng} \mathrm{m}^{-3}\right)\end{array}$} & CAMchem & - & 37.49 & 6.90 & 11.77 & 8.56 \\
\hline & CHASER_re1 & 37.49 & - & 34.45 & 28.24 & 45.76 \\
\hline & GEOSCHEM & 6.90 & 34.45 & - & 8.09 & 11.31 \\
\hline & GOCART-v5 & 11.77 & 28.24 & 8.09 & - & 19.29 \\
\hline & Oslo CTM3-v2 & 8.56 & 45.76 & 11.31 & 19.29 & - \\
\hline
\end{tabular}

63

64 (c) Tiksi

\begin{tabular}{|c|c|c|c|c|c|c|}
\hline Parameters & & CAMchem & CHASER_re1 & GEOSCHEM & GOCART-v5 & Oslo CTM3-v2 \\
\hline \multirow{5}{*}{$\mathrm{COR}^{\mathrm{a}}$} & CAMchem & - & 0.45 & 0.47 & 0.33 & 0.54 \\
\hline & CHASER_re1 & 0.45 & - & 0.78 & 0.77 & 0.64 \\
\hline & GEOSCHEM & 0.47 & 0.78 & - & 0.93 & 0.87 \\
\hline & GOCART-v5 & 0.33 & 0.77 & 0.93 & - & 0.75 \\
\hline & Oslo CTM3-v2 & 0.54 & 0.64 & 0.87 & 0.75 & - \\
\hline \multirow{5}{*}{$\begin{array}{c}\mathrm{NMB}^{\mathrm{b}} \\
(\%)\end{array}$} & CAMchem & - & -84.42 & -35.64 & -46.60 & 11.07 \\
\hline & CHASER_re1 & 813.44 & - & 310.05 & 240.81 & 633.13 \\
\hline & GEOSCHEM & 176.14 & -70.09 & - & -10.18 & 98.37 \\
\hline & GOCART-v5 & 200.57 & -67.11 & 18.35 & - & 121.63 \\
\hline & Oslo CTM3-v2 & 37.84 & -84.35 & -40.00 & -50.66 & - \\
\hline \multirow{5}{*}{$\begin{array}{c}\mathrm{NME}^{\mathrm{c}} \\
(\%)\end{array}$} & CAMchem & - & 84.42 & 54.38 & 57.93 & 60.09 \\
\hline & CHASER_re1 & 813.44 & - & 310.05 & 240.81 & 633.13 \\
\hline & GEOSCHEM & 186.23 & 70.09 & - & 20.65 & 104.94 \\
\hline & GOCART-v5 & 209.03 & 67.11 & 26.28 & - & 121.63 \\
\hline & Oslo CTM3-v2 & 67.46 & 84.35 & 50.86 & 50.66 & - \\
\hline \multirow{5}{*}{$\begin{array}{c}\mathrm{MB}^{\mathrm{d}} \\
\left(\mathrm{ng} \mathrm{m}^{-3}\right)\end{array}$} & CAMchem & - & -37.12 & -6.99 & -7.66 & 0.12 \\
\hline & CHASER_re1 & 37.12 & - & 30.14 & 29.46 & 37.24 \\
\hline & GEOSCHEM & 6.99 & -30.14 & - & -0.68 & 7.10 \\
\hline & GOCART-v5 & 7.66 & -29.46 & 0.68 & - & 7.78 \\
\hline & Oslo CTM3-v2 & -0.12 & -37.24 & -7.10 & -7.78 & - \\
\hline \multirow{2}{*}{$\begin{array}{c}\mathrm{MAE}^{\mathrm{e}} \\
\left(\mathrm{ng} \mathrm{m}^{-3}\right)\end{array}$} & CAMchem & - & 37.12 & 7.55 & 8.94 & 4.10 \\
\hline & CHASER_re1 & 37.12 & - & 30.14 & 29.46 & 37.24 \\
\hline
\end{tabular}




\begin{tabular}{cccccc} 
GEOSCHEM & 7.55 & 30.14 & - & 2.57 & 7.58 \\
\hline GOCART-v5 & 8.94 & 29.46 & 2.57 & - & 7.78 \\
\hline Oslo CTM3-v2 & 4.10 & 37.24 & 7.58 & 7.78 & -
\end{tabular}

65

66 (d) Zeppelin

\begin{tabular}{|c|c|c|c|c|c|c|}
\hline Parameters & & CAMchem & CHASER_re1 & GEOSCHEM & GOCART-v5 & Oslo CTM3-v2 \\
\hline \multirow{5}{*}{$\mathrm{COR}^{\mathrm{a}}$} & CAMchem & - & 0.72 & 0.88 & 0.93 & 0.57 \\
\hline & CHASER_re1 & 0.72 & - & 0.51 & 0.72 & 0.61 \\
\hline & GEOSCHEM & 0.88 & 0.51 & - & 0.92 & 0.57 \\
\hline & GOCART-v5 & 0.93 & 0.72 & 0.92 & - & 0.76 \\
\hline & Oslo CTM3-v2 & 0.57 & 0.61 & 0.57 & 0.76 & - \\
\hline \multirow{5}{*}{$\begin{array}{c}\mathrm{NMB}^{\mathrm{b}} \\
(\%)\end{array}$} & CAMchem & - & -89.52 & -66.10 & -77.05 & 61.88 \\
\hline & CHASER_re1 & 1116.80 & - & 310.46 & 167.04 & 1389.68 \\
\hline & GEOSCHEM & 230.01 & -65.56 & - & -29.68 & 408.70 \\
\hline & GOCART-v5 & 378.64 & -53.57 & 50.21 & - & 520.07 \\
\hline & Oslo CTM3-v2 & 44.23 & -87.53 & -58.64 & -73.17 & - \\
\hline \multirow{5}{*}{$\begin{array}{c}\mathrm{NME}^{\mathrm{c}} \\
(\%)\end{array}$} & CAMchem & - & 89.52 & 66.10 & 77.05 & 108.27 \\
\hline & CHASER_re1 & 1116.80 & - & 310.46 & 167.04 & 1389.68 \\
\hline & GEOSCHEM & 230.01 & 65.56 & - & 32.28 & 408.70 \\
\hline & GOCART-v5 & 378.64 & 53.57 & 52.47 & - & 520.07 \\
\hline & Oslo CTM3-v2 & 81.19 & 87.53 & 58.64 & 73.17 & - \\
\hline \multirow{5}{*}{$\begin{array}{c}\mathrm{MB}^{\mathrm{d}} \\
\left(\mathrm{ng} \mathrm{m}^{-3}\right)\end{array}$} & CAMchem & - & -27.11 & -6.81 & -10.72 & -0.48 \\
\hline & CHASER_re1 & 27.11 & - & 20.30 & 16.40 & 26.63 \\
\hline & GEOSCHEM & 6.81 & -20.30 & - & -3.91 & 6.33 \\
\hline & GOCART-v5 & 10.72 & -16.40 & 3.91 & - & 10.24 \\
\hline & Oslo CTM3-v2 & 0.48 & -26.63 & -6.33 & -10.24 & - \\
\hline \multirow{5}{*}{$\begin{array}{c}\mathrm{MAE}^{\mathrm{e}} \\
\left(\mathrm{ng} \mathrm{m}^{-3}\right)\end{array}$} & CAMchem & - & 27.11 & 6.81 & 10.72 & 1.84 \\
\hline & CHASER_re1 & 27.11 & - & 20.30 & 16.40 & 26.63 \\
\hline & GEOSCHEM & 6.81 & 20.30 & - & 4.27 & 6.33 \\
\hline & GOCART-v5 & 10.72 & 16.40 & 4.27 & - & 10.24 \\
\hline & Oslo CTM3-v2 & 1.84 & 26.63 & 6.33 & 10.24 & - \\
\hline
\end{tabular}

67

${ }^{a}$ Correlative coefficient. ${ }^{b}$ Normalized mean bias. ${ }^{\mathrm{c}}$ Normalized mean error. ${ }^{\mathrm{d}}$ Mean bias. ${ }^{\mathrm{e}}$ Mean absolute error. 
(a)
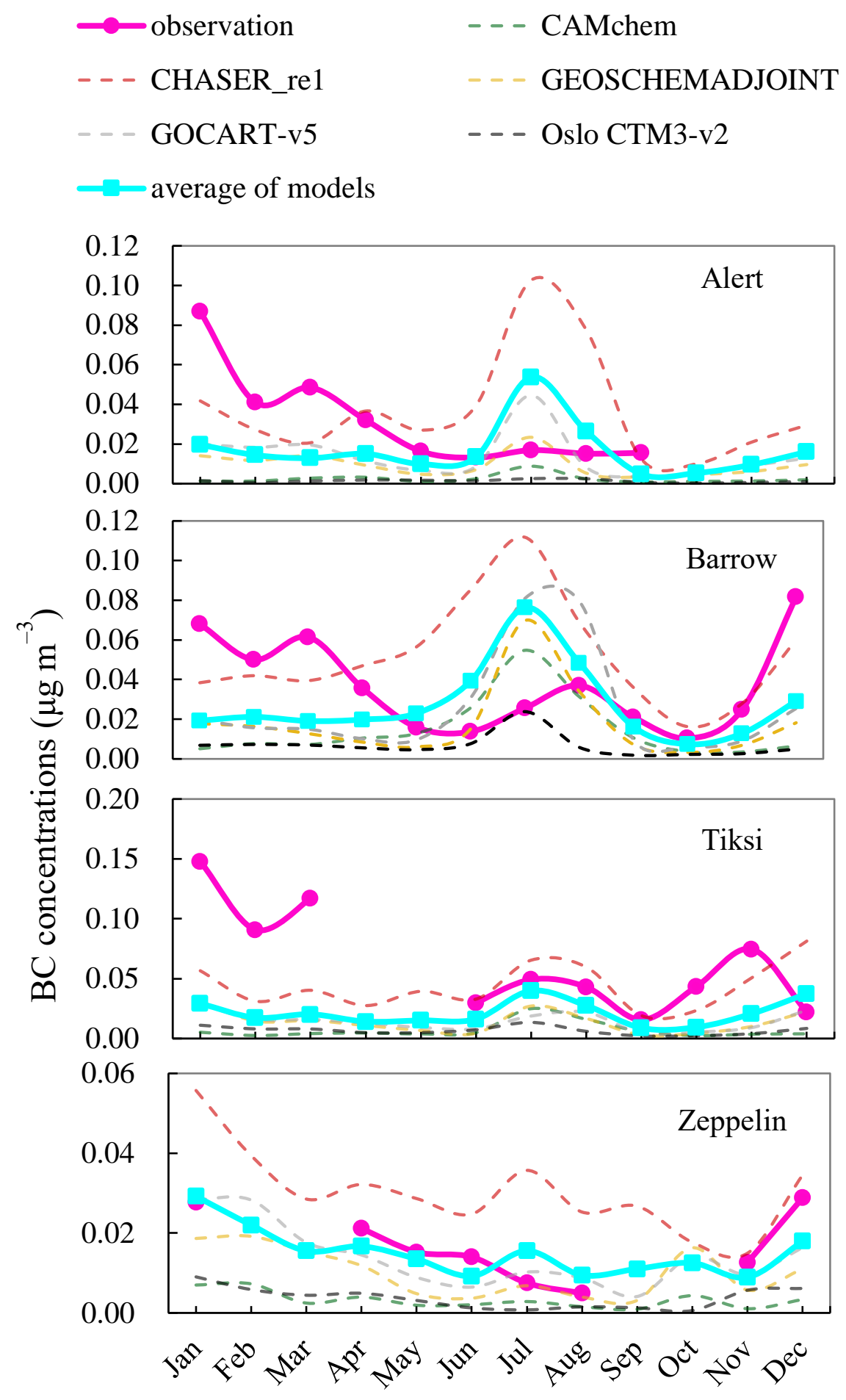


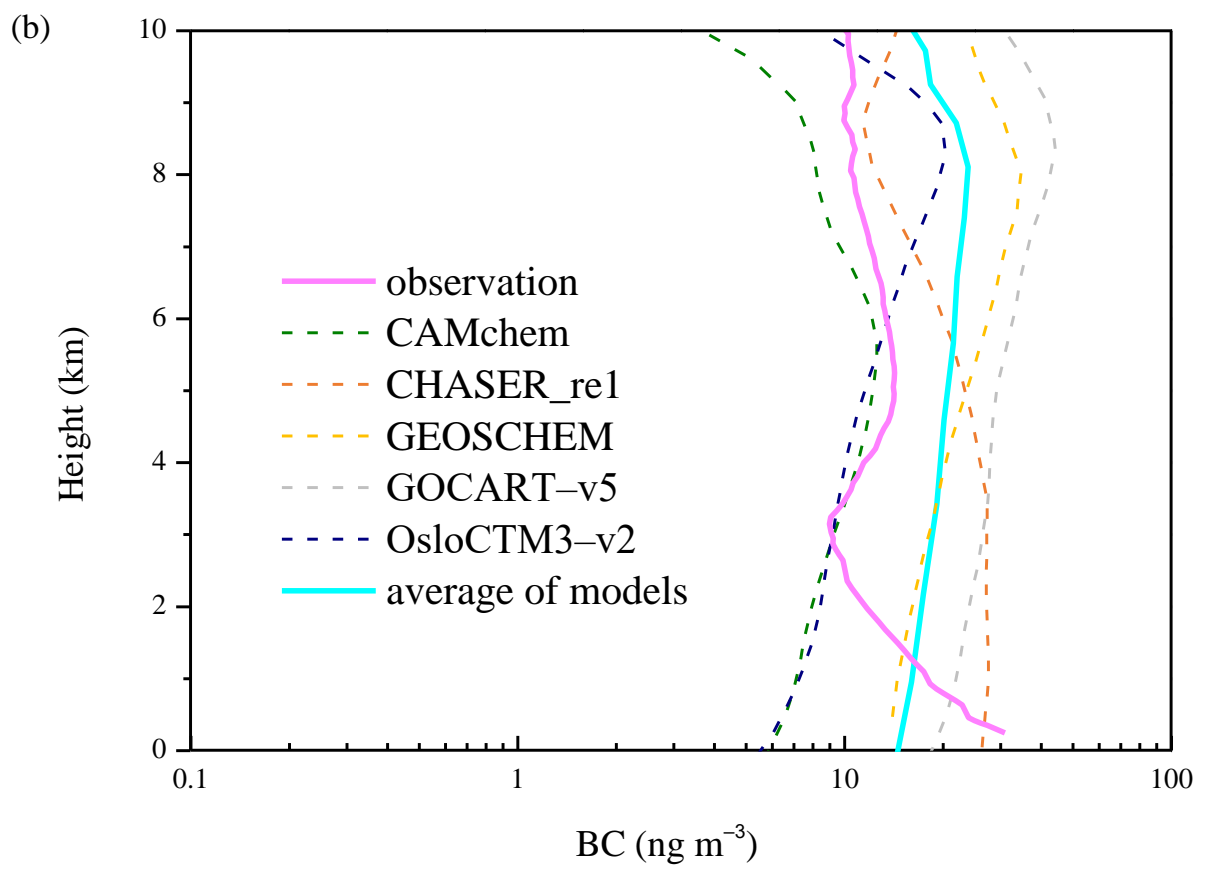

72 Figure S2 (a) Temporal variations in simulated (BASE scenario) and observed BC concentrations near 73 surface at Alert, Barrow, Tiks, and Zeppelin in 2010. (b) Comparison between the vertical profiles of 74 simulated (BASE simulation) and observed BC concentrations (HIPPO) during 24 March-16 April 752010.

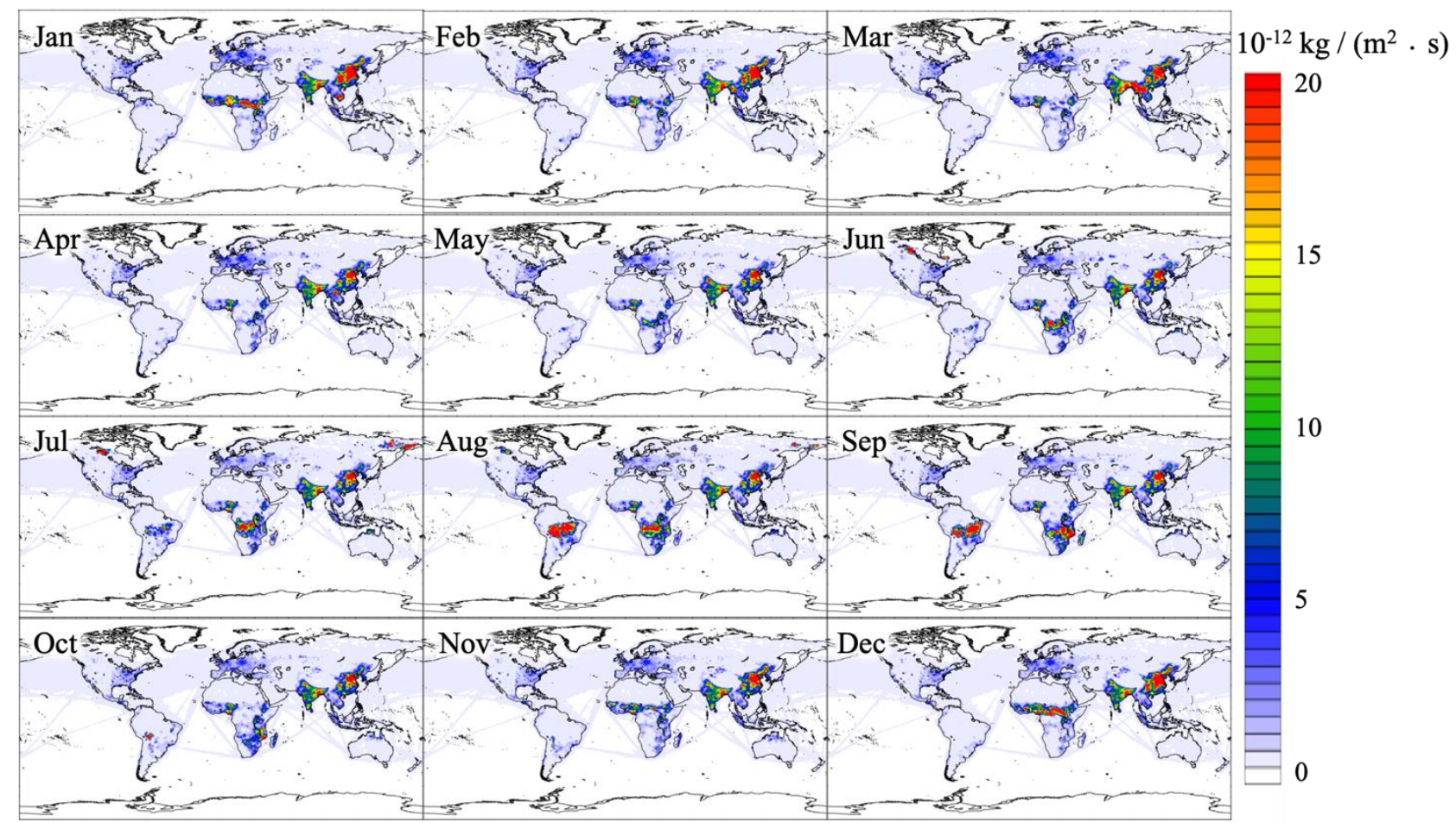

Figure S3. Spatial distribution of monthly BC emissions in 2010. 


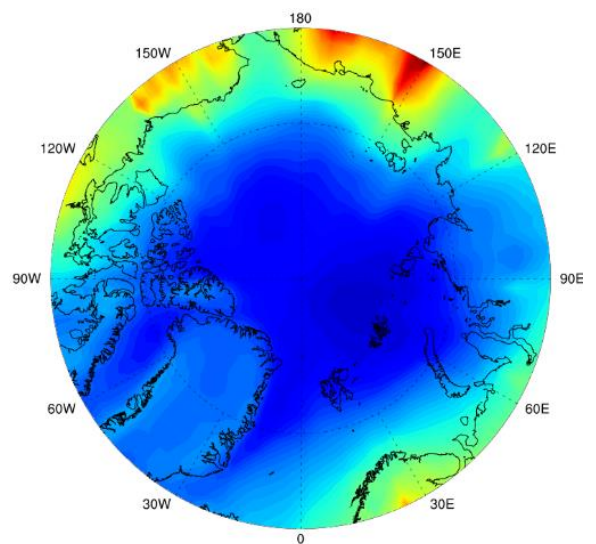

(a) CAMchem - summer

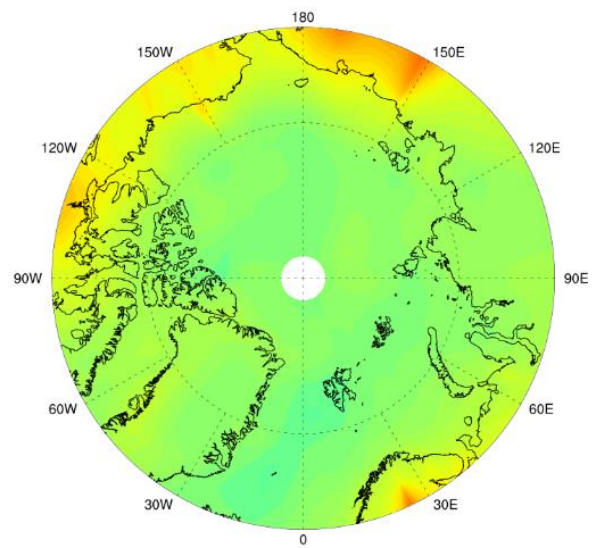

(c) CHASER_re1 - summer

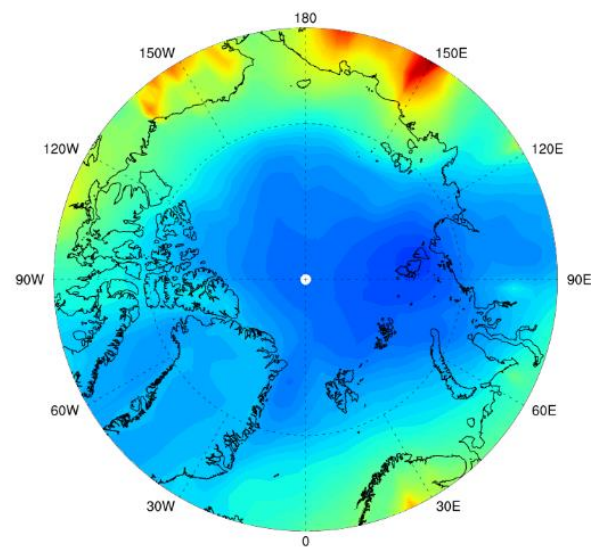

(e) GEOS-chem - summer

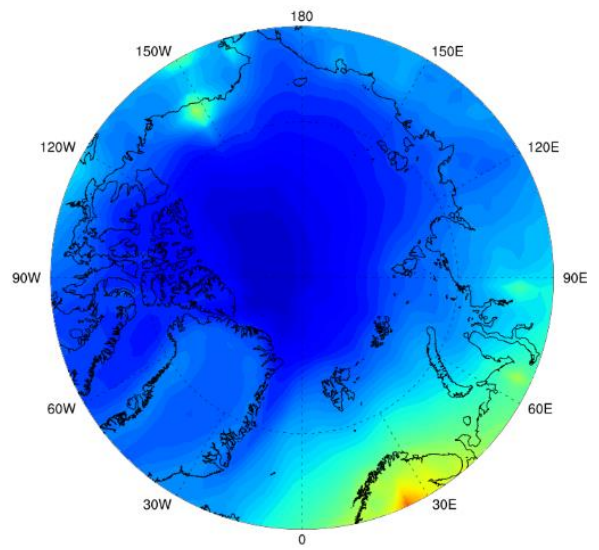

(b) CAMchem - winter

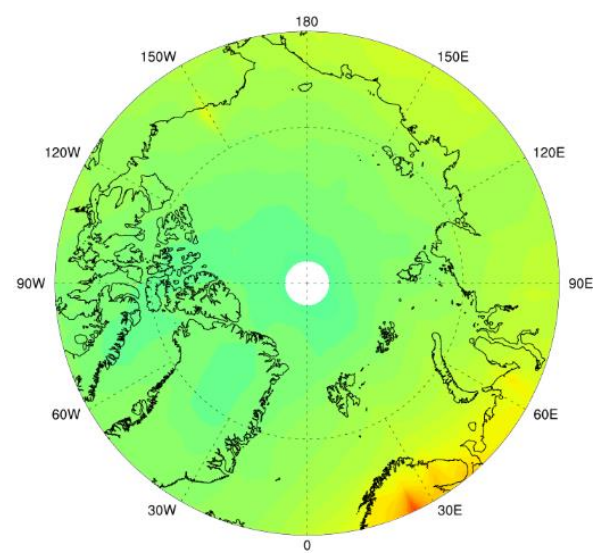

(d) CHASER_re1 - winter

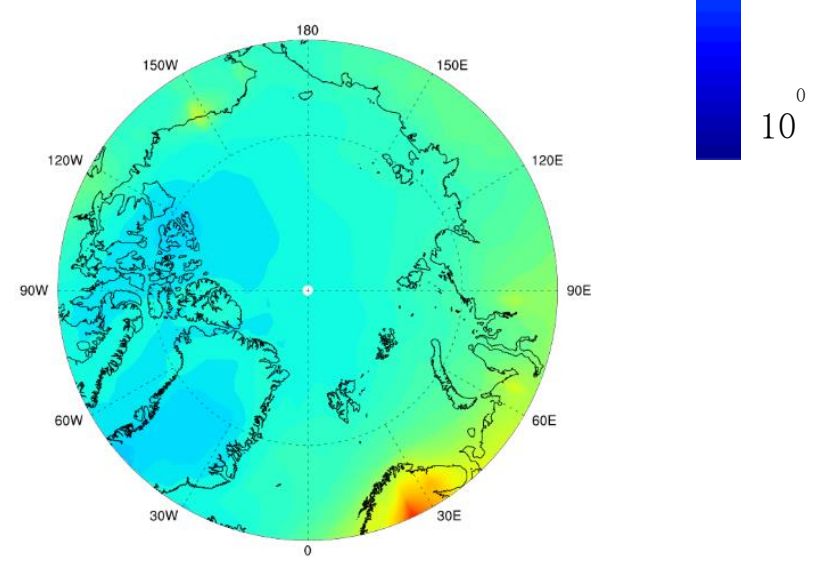

(f) GEOS-chem - winter ng m

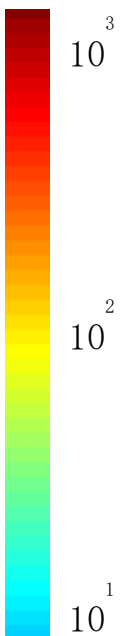

10

0 


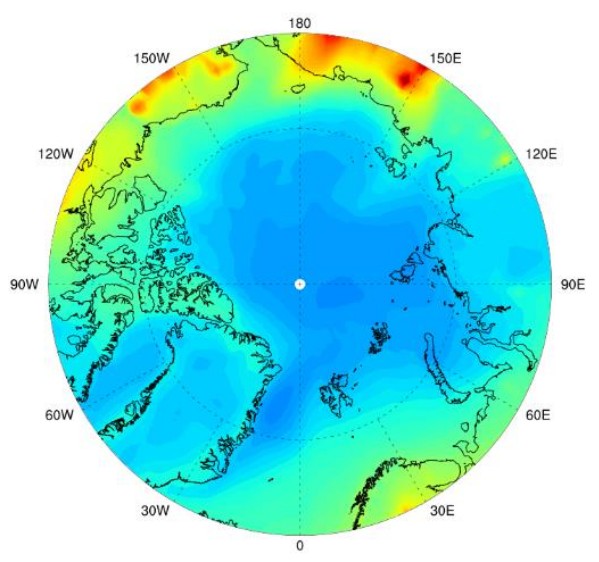

(g) GOCART-v5 - summer

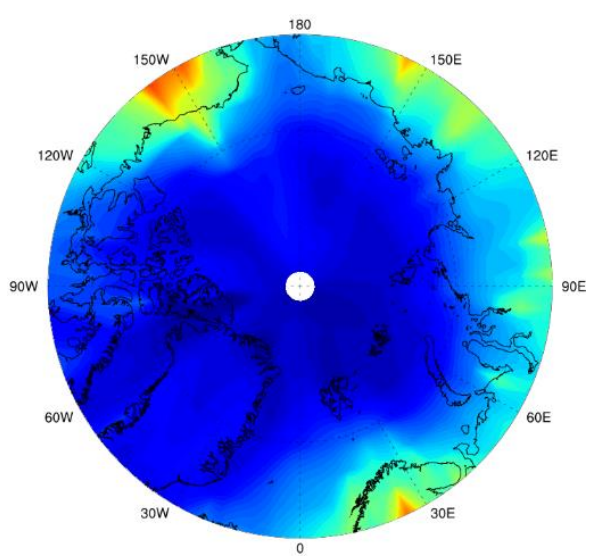

(i) Oslo CTM3-v2 - summer

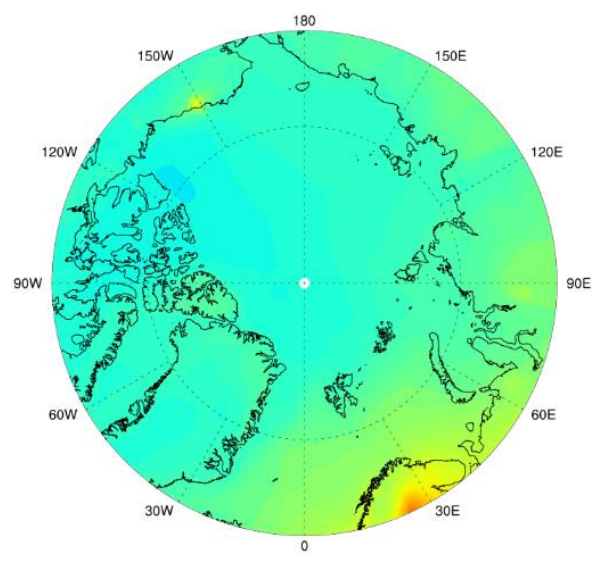

(h) GOCART-v5 - winter

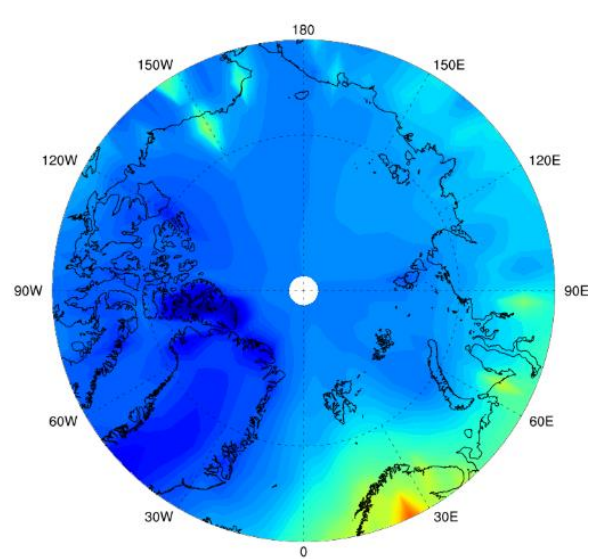

(j) Oslo CTM3-v2 - winter

80 Figure S4. Spatial distributions of BC concentrations for each model in different seasons (summer and 81 winter) in the Arctic near surface in 2010. 


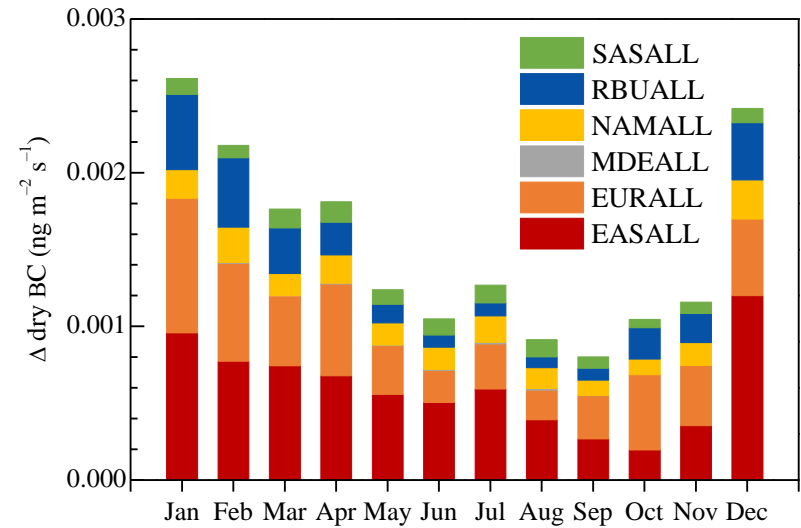

(a)

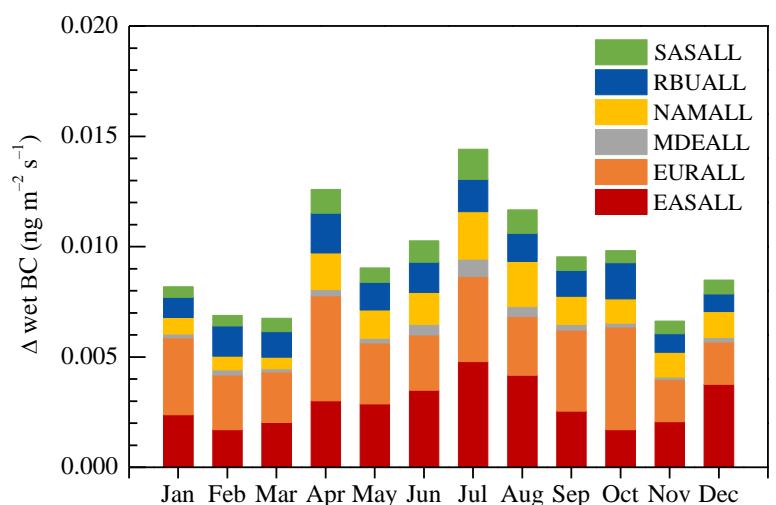

(b)

85 Figure S5. Monthly mean reduced (a) dry and (b) wet depositions of the near surface Arctic BC due 86 to $20 \%$ emission reductions of six source regions in 2010.

87 


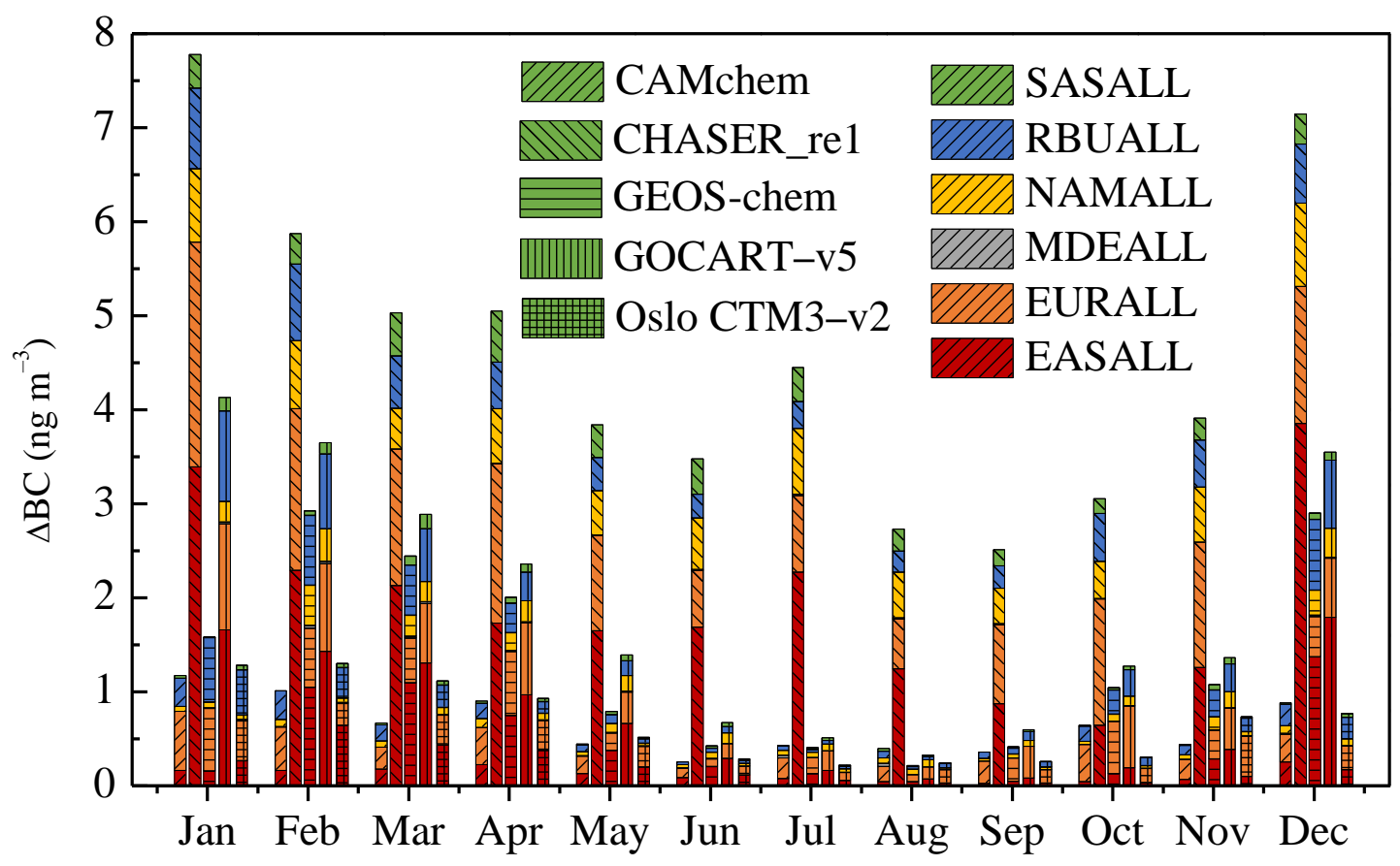

89

90 Figure S6. Monthly reduced concentrations of the near-surface Arctic BC due to $20 \%$ emission 91 reductions from six source regions for each model in 2010.

92 
Table S2 The vertical stratification unified according to the pressure of CHASER_re1

\begin{tabular}{|c|c|c|c|c|c|c|c|c|c|c|c|c|c|c|c|c|c|c|c|c|c|c|}
\hline \multicolumn{2}{|c|}{ The unified layers of this study } & 1 & 2 & 3 & 4 & 5 & 6 & 7 & 8 & 9 & 10 & 11 & 12 & 13 & 14 & 15 & 16 & 17 & 18 & 19 & 20 & 21 \\
\hline \multirow{2}{*}{\multicolumn{2}{|c|}{ Pressure $(\mathrm{kPa})$}} & $>$ & $97-$ & $92-$ & $85-$ & $78-$ & $68-$ & $60-$ & $52-$ & $45-$ & $39-$ & $34-$ & $30-$ & $26-$ & $23-$ & $20-$ & $17-$ & $15-$ & $13-$ & $11.5-$ & $10-$ & $<10$ \\
\hline & & 99.5 & 99.5 & 97 & 92 & 85 & 78 & 68 & 60 & 52 & 45 & 39 & 34 & 30 & 26 & 23 & 20 & 17 & 15 & 13 & 11.5 & \\
\hline \multirow{10}{*}{$\begin{array}{l}\text { The original } \\
\text { layers of } \\
\text { participating } \\
\text { models }\end{array}$} & \multirow[t]{2}{*}{ CAMchem } & \multirow[t]{2}{*}{1} & \multirow[t]{2}{*}{$2-3$} & \multirow[t]{2}{*}{$4-6$} & $7-$ & $12-$ & $15-$ & $19-$ & $21-$ & $23-$ & $25-$ & \multirow[t]{2}{*}{27} & \multirow[t]{2}{*}{28} & \multirow[t]{2}{*}{29} & \multirow[t]{2}{*}{$-^{\mathrm{a}}$} & \multirow[t]{2}{*}{30} & \multirow[t]{2}{*}{31} & \multirow[t]{2}{*}{32} & \multirow[t]{2}{*}{33} & \multirow[t]{2}{*}{34} & \multirow[t]{2}{*}{35} & $36-$ \\
\hline & & & & & 11 & 14 & 18 & 20 & 22 & 34 & 26 & & & & & & & & & & & 56 \\
\hline & \multirow[t]{2}{*}{ CHASER_re1 } & \multirow[t]{2}{*}{1} & \multirow[t]{2}{*}{2} & \multirow[t]{2}{*}{3} & \multirow[t]{2}{*}{4} & \multirow[t]{2}{*}{5} & \multirow[t]{2}{*}{6} & \multirow[t]{2}{*}{7} & \multirow[t]{2}{*}{8} & \multirow[t]{2}{*}{9} & \multirow[t]{2}{*}{10} & \multirow[t]{2}{*}{11} & \multirow[t]{2}{*}{12} & \multirow[t]{2}{*}{13} & 14 & 15 & 16 & 17 & 18 & 19 & 20 & $21-$ \\
\hline & & & & & & & & & & & & & & & & & & & & & & 32 \\
\hline & GEOSCHEM & $1-2$ & 3 & $4-7$ & $8-$ & $12-$ & $16-$ & $20-$ & $22-$ & 24 & 26 & 27 & 28 & 29 & 30 & 31 & 32 & 33 & $-{ }^{a}$ & 34 & 35 & $36-$ \\
\hline & & & & & 11 & 15 & 19 & 21 & 23 & 25 & & & & & & & & & & & & 47 \\
\hline & GOCART-v5 & 1 & $2-3$ & $4-6$ & $7-$ & $12-$ & $15-$ & $19-$ & $21-$ & $23-$ & $25-$ & 27 & 28 & 29 & $-{ }^{a}$ & 30 & 31 & 32 & 33 & 34 & 35 & $36-$ \\
\hline & & & & & 11 & 14 & 18 & 20 & 22 & 34 & 26 & & & & & & & & & & & 72 \\
\hline & OsloCTM3-v2 & $1-2$ & $3-5$ & $6-$ & $10-$ & 13 & $15-$ & $18-$ & $20-$ & $22-$ & 24 & $25-$ & 27 & 28 & 29 & 30 & 32 & 33 & 34 & $34-$ & 35 & $36-$ \\
\hline & & & & 9 & 12 & 14 & 17 & 19 & 21 & 23 & & 26 & & & & 31 & & & & 35 & & 60 \\
\hline
\end{tabular}

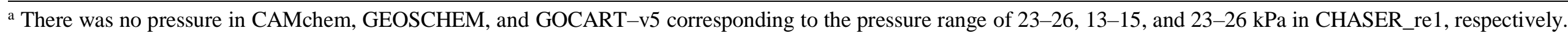
The BC concentrations were calculated from the average of concentrations of two adjacent layers. 


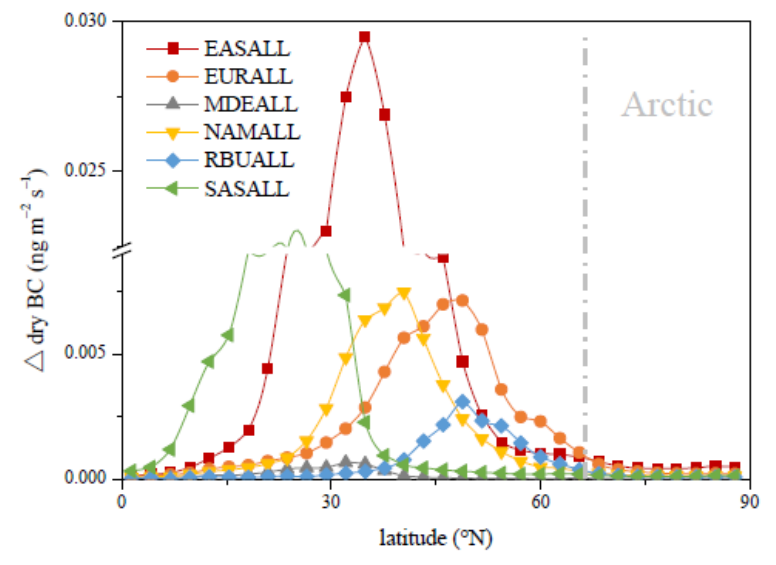

(a) dry deposition - summer

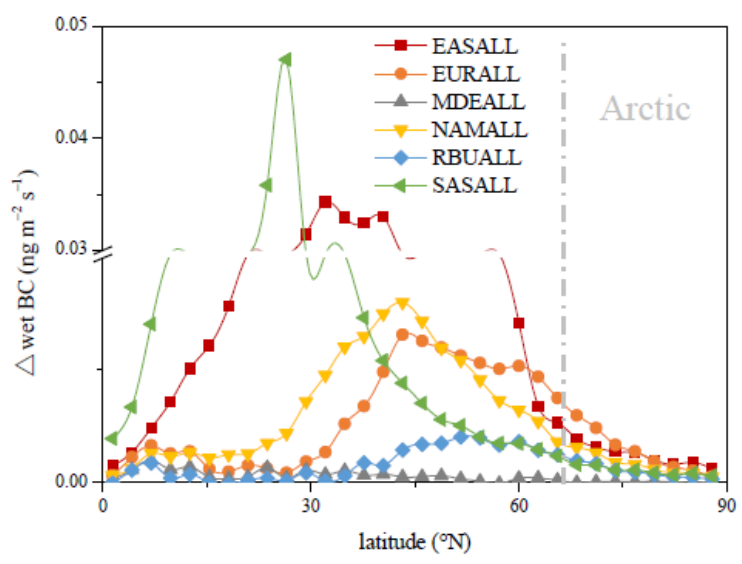

(c) wet deposition - summer

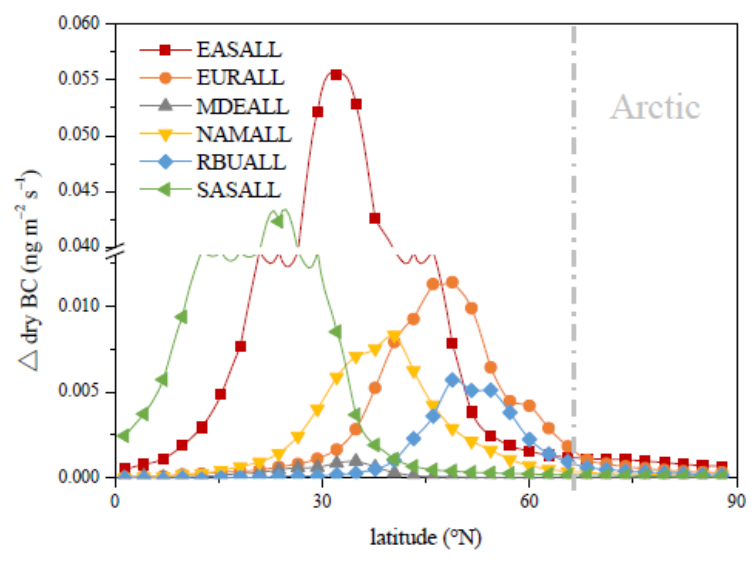

(b) dry deposition - winter

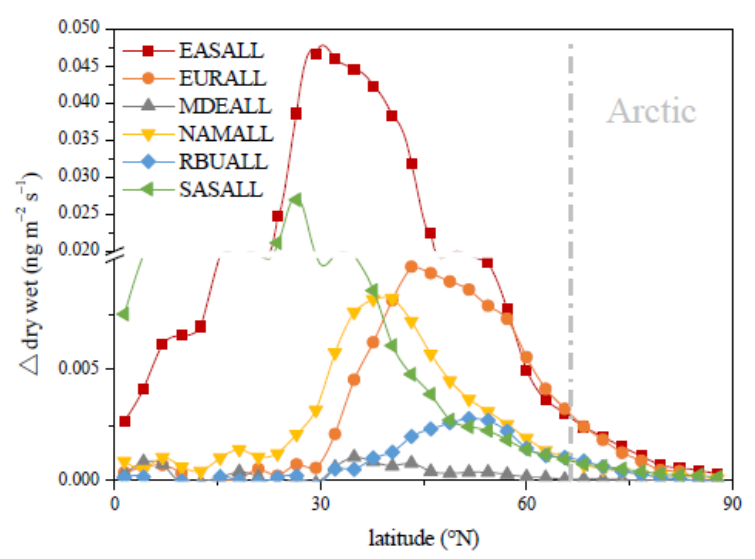

(d) wet deposition - winter

Figure S7. Reduced dry and wet depositions of the near surface Northern Hemisphere BC due to 20\% emission reductions from six source regions during summer and winter in 2010. 


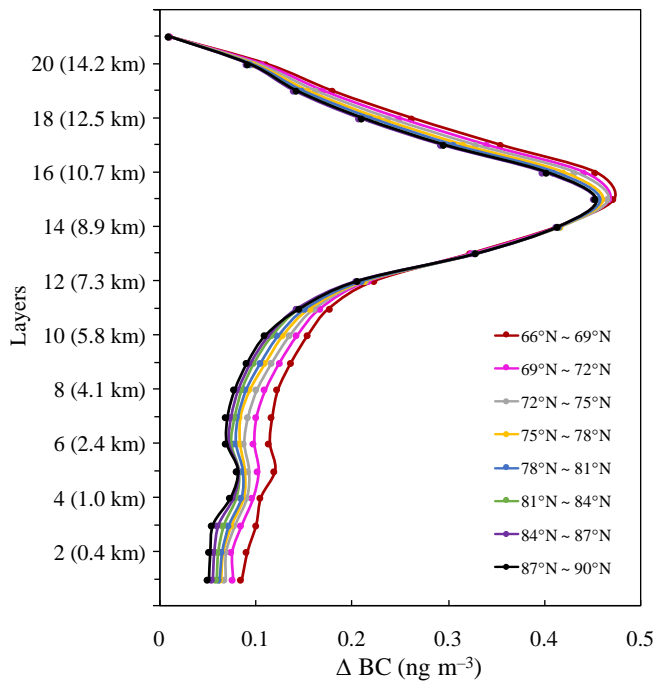

(a)

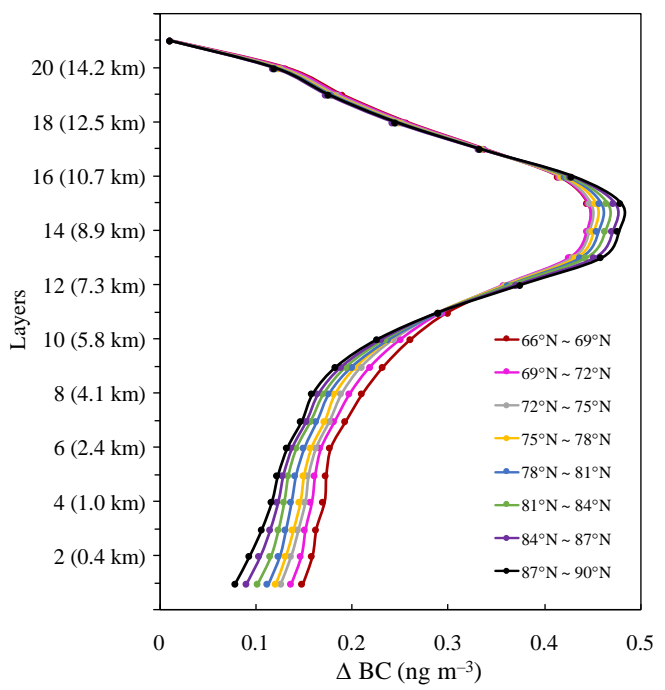

(b)

Figure S8. Contributions of $20 \%$ emission perturbation in SAS to BC concentrations of Arctic in different latitude bands varies with altitude in (a) summer and (b) winter in 2010. 